Pacific

Journal of

Mathematics

\title{
COMMENSURATIONS AND METRIC PROPERTIES OF HOUGHTON'S GROUPS
}

José Burillo, Sean Cleary, Armando Martino and ClaAs E. Röver 


\title{
COMMENSURATIONS AND METRIC PROPERTIES OF HOUGHTON'S GROUPS
}

\author{
José Burillo, Sean Cleary, Armando Martino and ClaAs E. Röver
}

\begin{abstract}
We describe the automorphism groups and the abstract commensurators of Houghton's groups. Then we give sharp estimates for the word metric of these groups and deduce that the commensurators embed into the corresponding quasi-isometry groups. As a further consequence, we obtain that the Houghton group on two rays is at least quadratically distorted in those with three or more rays.
\end{abstract}

\section{Introduction}

The Houghton groups $\mathcal{H}_{n}$, introduced in [Houghton 1978], form an interesting family whose homological finiteness properties were described in [Brown 1987]. Röver [1999] showed that the $\mathcal{H}_{n}$ are all subgroups of Thompson's group $V$, and Lehnert [2009] described the metric for $\mathcal{H}_{2}$. Lee [2012] described isoperimetric bounds, and de Cornulier, Guyot and Pitsch [CGP 2007] showed that they are isolated points in the space of groups.

Here, we classify automorphisms and determine the abstract commensurator of $\mathcal{H}_{n}$. We also give sharp estimates for the word metric which are sufficient to show that the map from the abstract commensurator to the group of quasi-isometries of $\mathcal{H}_{n}$ is an injection.

\section{Definitions and background}

Let $\mathbb{N}$ be the set of natural numbers (positive integers) and $n \geq 1$ be an integer. We write $\mathbb{Z}_{n}$ for the integers modulo $n$ with addition and put $R_{n}=\mathbb{Z}_{n} \times \mathbb{N}$. We interpret $R_{n}$ as the graph of $n$ pairwise disjoint rays; each vertex $(i, k)$ is connected to $(i, k+1)$. We denote by Sym $_{n}, \mathrm{FSym}_{n}$ and FAlt ${ }_{n}$, or simply Sym, FSym and FAlt if $n$ is understood, the full symmetric group, the finitary symmetric group and the finitary alternating group on the set $R_{n}$, respectively.

The authors thank Charles Cox for his remarks on an earlier version of this paper and NUI Galway for its hospitality. This work was partially supported by MEC grant \#MTM2011-25955 (Burillo), National Science Foundation grant \#1417820 (Cleary) and Simons Foundation grant \#234548 (Cleary).

MSC2010: 20F65.

Keywords: Houghton's groups, commensurations. 
The Houghton group $\mathcal{H}_{n}$ is the subgroup of Sym consisting of those permutations that are eventually translations (of each of the rays). In other words, the permutation $\sigma$ of the set $R_{n}$ is in $\mathcal{H}_{n}$ if there exist integers $N \geq 0$ and $t_{i}=t_{i}(\sigma)$ for $i \in \mathbb{Z}_{n}$ such that for all $k \geq N$, we have $(i, k) \sigma=\left(i, k+t_{i}\right)$; throughout we will use right actions.

Note that necessarily the sum of the translations $t_{i}$ must be zero because the permutation needs of course to be a bijection. This implies that $\mathcal{H}_{1} \cong$ FSym.

For $i, j \in \mathbb{Z}_{n}$ with $i \neq j$ let $g_{i j} \in \mathcal{H}_{n}$ be the element which translates the line obtained by joining rays $i$ and $j$, given by

$$
\begin{aligned}
& (i, n) g_{i j}=(i, n-1) \quad \text { if } n>1 \text {, } \\
& (i, 1) g_{i j}=(j, 1) \text {, } \\
& (j, n) g_{i j}=(j, n+1) \quad \text { if } n \geq 1 \text {, and } \\
& (k, n) g_{i j}=(k, n) \quad \text { if } k \notin\{i, j\} .
\end{aligned}
$$

We also write $g_{i}$ instead of $g_{i i+1}$. It is easy to see that $\left\{g_{i} \mid i \in \mathbb{Z}_{n}\right\}$, and $\left\{g_{i j} \mid i, j \in \mathbb{Z}_{n}, i \neq j\right\}$, are generating sets for $\mathcal{H}_{n}$ if $n \geq 3$ as we can simply check that the commutator $\left[g_{0}, g_{1}\right]=g_{0}^{-1} g_{1}^{-1} g_{0} g_{1}$ transposes $(1,1)$ and $(2,1)$. In the special case of $\mathcal{H}_{2}$, the element $g_{1}$ is redundant as $g_{1}=g_{0}^{-1}$. Further, an additional generator to $g_{0}$ is required to generate the group; we choose $\tau$ which fixes all points except for transposing $(0,1)$ and $(1,1)$.

It is now clear that the commutator subgroup of $\mathcal{H}_{n}$ is given by

$$
\mathcal{H}_{n}^{\prime}= \begin{cases}\text { FAlt } & \text { if } n \leq 2 \\ \text { FSym } & \text { if } n \geq 3\end{cases}
$$

For $n \geq 3$, we thus have a short exact sequence

$$
1 \rightarrow \mathrm{FSym} \rightarrow \mathcal{H}_{n} \stackrel{\pi}{\longrightarrow} \mathbb{Z}^{n-1} \rightarrow 1,
$$

where $\pi(\sigma)=\left(t_{0}(\sigma), \ldots, t_{n-2}(\sigma)\right)$ is the abelianization homomorphism. We note that as the sum of all the eventual translations must be zero, the last translation is determined by the preceding ones:

$$
t_{n-1}(\sigma)=-\sum_{i=0}^{n-2} t_{i}(\sigma) .
$$

We will use the following facts freely throughout this paper; see [Dixon and Mortimer 1996] or [Scott 1964].

Lemma 1.1. The group FAlt is simple and equal to the commutator subgroup of FSym, and Aut (FAlt $)=\operatorname{Aut}($ FSym $)=$ Sym. 


\section{Automorphisms of $\mathcal{H}_{n}$}

Here we determine the automorphism group of $\mathcal{H}_{n}$. First we establish that we have to look no further than Sym. We let $N_{G}(H)$ denote the normalizer, in $G$, of the subgroup $H$ of $G$.

Proposition 2.1. Every automorphism of $\mathcal{H}_{n}, n \geq 1$, is given by conjugation by an element of Sym; that is to say, $\operatorname{Aut}\left(\mathcal{H}_{n}\right)=N_{\mathrm{Sym}}\left(\mathcal{H}_{n}\right)$.

Proof. From the above, the finitary alternating group FAlt is the second derived subgroup of $\mathcal{H}_{n}$, and hence characteristic in $\mathcal{H}_{n}$. So every automorphism of $\mathcal{H}_{n}$ restricts to an automorphism of FAlt. Since Aut(FAlt) $=$ Sym, this restriction yields a homomorphism $\operatorname{Aut}\left(\mathcal{H}_{n}\right) \rightarrow$ Sym and we need to show that it is injective with image equal to $N_{\text {Sym }}\left(\mathcal{H}_{n}\right)$.

In order to see this let $\psi \in \operatorname{Aut}\left(\mathcal{H}_{n}\right)$ be an automorphism. Compose this with an inner automorphism (of Sym) so that the result is an (injective) homomorphism $\alpha: \mathcal{H}_{n} \rightarrow$ Sym whose restriction to FAlt is trivial. We let $k \in \mathbb{N}$ and consider the six consecutive points $a_{\ell}=(i, k+\ell)$ of $R_{n}$ for $\ell \in\{0,1, \ldots, 5\}$.

We denote by $g_{i}^{\alpha}$ the image of $g_{i}$ under $\alpha$, and by $\left(\begin{array}{lll}x y z & z\end{array}\right)$ the 3 -cycle of the points $x, y$ and $z$. Using the identities

$$
g_{i}^{-1}\left(a_{1} a_{2} a_{3}\right) g_{i}=\left(a_{0} a_{1} a_{2}\right) \quad \text { and } \quad g_{i}^{-1}\left(a_{3} a_{4} a_{5}\right) g_{i}=\left(a_{2} a_{3} a_{4}\right)
$$

and applying $\alpha$, which is trivial on FAlt, we get

$$
\left(g_{i}^{\alpha}\right)^{-1}\left(a_{1} a_{2} a_{3}\right) g_{i}^{\alpha}=\left(a_{0} a_{1} a_{2}\right) \quad \text { and } \quad\left(g_{i}^{\alpha}\right)^{-1}\left(a_{3} a_{4} a_{5}\right) g_{i}^{\alpha}=\left(a_{2} a_{3} a_{4}\right) .
$$

Hence, $g_{i}^{\alpha}$ maps $\left\{a_{1}, a_{2}, a_{3}\right\}$ to $\left\{a_{0}, a_{1}, a_{2}\right\}$, and then also $\left\{a_{3}, a_{4}, a_{5}\right\}$ to $\left\{a_{2}, a_{3}, a_{4}\right\}$. The conclusion is that it maps $a_{3}$ to $a_{2}$. Applying a similar argument to all points in the branches $i$ and $i+1$, it follows that $g_{i}^{\alpha}=g_{i}$, and since $i$ was arbitrary, this means that $\alpha$ is the identity map.

With Lemma 1.1 in mind we now present the complete description of $\operatorname{Aut}\left(\mathcal{H}_{n}\right)$.

Theorem 2.2. For $n \geq 2$, the automorphism group $\operatorname{Aut}\left(\mathcal{H}_{n}\right)$ of the Houghton group $\mathcal{H}_{n}$ is isomorphic to the semidirect product $\mathcal{H}_{n} \rtimes \mathcal{S}_{n}$, where $\mathcal{S}_{n}$ is the symmetric group that permutes the $n$ rays.

Proof. By the proposition, it suffices to prove that every $\alpha \in$ Sym which normalizes $\mathcal{H}_{n}$ must map $(i, k+m)$ to $(j, l+m)$ for some $k, l \geq 1$ and all $m \geq 0$.

To this end, we pick $\alpha \in N_{\text {Sym }}\left(\mathcal{H}_{n}\right)$ and $\sigma \in \mathcal{H}_{n}$. Since $\sigma^{\alpha}\left(=\alpha^{-1} \sigma \alpha\right)$ is in $\mathcal{H}_{n}$ and maps the point $x \alpha$ to $(x \sigma) \alpha$, these two points must lie on the same ray for all but finitely many $x \in R_{n}$. Similarly, $x$ and $x \sigma$ lie on the same ray for all but finitely many $x \in R_{n}$, as $\sigma \in \mathcal{H}_{n}$. In fact, given a ray, we can choose $\sigma$ so that whenever $x$ lies on that ray, $x$ and $x \sigma$ are successors on the same ray. Combining these facts, we see that $\alpha$ maps all but finitely many points of ray $i$ to one and the same ray, say ray $j$. 
This defines a homomorphism from $\operatorname{Aut}\left(\mathcal{H}_{n}\right)$ onto $\mathcal{S}_{n}$, which is obviously split, since given a permutation of the $n$ rays, it clearly defines an automorphism of $\mathcal{H}_{n}$.

So assume that $\alpha$ is now in the kernel of that map, so it does not permute the rays, and take $\sigma$ to be a $g_{j i}$ generator of $\mathcal{H}_{n}$, i.e., an infinite cycle inside Sym. Then, since conjugating inside Sym preserves cycle types, the element $\sigma^{\alpha} \in \mathcal{H}_{n}$ is also a single infinite cycle. This means that $\sigma^{\alpha}$ has nonzero translations in only two rays, and these translations are 1 and -1 . Any point in the support of $\sigma$ is sent into the $i$-th ray by $\sigma^{k}$, for all sufficiently large $k$. Therefore, as $\alpha$ sends almost all points in the $i$-th ray into the $i$-th ray, the same is true for $\sigma^{\alpha}$. Hence $t_{i}\left(\sigma^{\alpha}\right)$ is positive, so it must be $t_{i}\left(\sigma^{\alpha}\right)=1$. It is quite clear now that $\alpha$ translates by an integer in the ray $i$, sufficiently far out. This finishes the proof since this could be done for any $i$, and hence $\alpha \in \mathcal{H}_{n}$.

\section{Commensurations of $\mathcal{H}_{n}$}

First, we recall that a commensuration of a group $G$ is an isomorphism $A \stackrel{\phi}{\longrightarrow} B$, where $A$ and $B$ are subgroups of finite index in $G$. Two commensurations $\phi$ and $\psi$ of $G$ are equivalent if there exists a subgroup $A$ of finite index in $G$ such that the restrictions of $\phi$ and $\psi$ to $A$ are equal. The set of all commensurations of $G$ modulo this equivalence relation forms a group, known as the (abstract) commensurator of $G$, and is denoted $\operatorname{Com}(G)$. In this section we will determine the commensurator of $\mathcal{H}_{n}$.

For a moment, we let $H$ be a subgroup of a group $G$. The relative commensurator of $H$ in $G$ is denoted $\operatorname{Com}_{G}(H)$ and consists of those $g \in G$ such that $H \cap H^{g}$ has finite index in both $H$ and $H^{g}$. Similar to the homomorphism from $N_{G}(H)$ to $\operatorname{Aut}(H)$, there is a homomorphism from $\operatorname{Com}_{G}(H)$ to $\operatorname{Com}(H)$; its kernel consists of those elements of $G$ that centralize a finite index subgroup of $H$.

To pin down $\operatorname{Com}\left(\mathcal{H}_{n}\right)$, we first establish that every commensuration of $\mathcal{H}_{n}$ can be seen as conjugation by an element of Sym, and then characterize $\operatorname{Com}_{\mathrm{Sym}}\left(\mathcal{H}_{n}\right)$.

Since a commensuration $\phi$ and its restriction to a subgroup of finite index in its domain are equivalent, we can restrict our attention to the following family of subgroups of finite index in $\mathcal{H}_{n}$, in order to exhibit $\operatorname{Com}\left(\mathcal{H}_{n}\right)$. For every integer $p \geq 1$, we define the subgroup $U_{p}$ of $\mathcal{H}_{n}$ by

$$
U_{p}=\left\langle\text { FAlt, } g_{i}^{p} \mid i \in \mathbb{Z}_{n}\right\rangle \text {. }
$$

We collect some useful properties of these subgroups first, where $A \subset_{f} B$ means that $A$ is a subgroup of finite index in $B$.

Lemma 3.1. Let $n \geq 3$.

(i) For every $p$, the group $U_{p}$ coincides with $\mathcal{H}_{n}^{p}$, the subgroup generated by all p-th powers in $\mathcal{H}_{n}$, and hence is characteristic in $\mathcal{H}_{n}$. 
(ii) $U_{p}^{\prime}=\left\{\begin{array}{ll}\text { FAlt, } & \text { p even, } \\ \text { FSym, } & \text { podd; }\end{array}\right.$ and $\left|\mathcal{H}_{n}: U_{p}\right|= \begin{cases}2 p^{n-1}, & p \text { even, } \\ p^{n-1}, & p \text { odd }\end{cases}$

(iii) For every finite index subgroup $U$ of $\mathcal{H}_{n}$, there exists a $p \geq 1$ with FAlt $=$ $U_{p}^{\prime} \subset U_{p} \subset{ }_{f} U \subset_{f} \mathcal{H}_{n}$.

The same is essentially true for the case $n=2$, except that $U_{p}^{\prime}$ is always equal to FAlt in this case, with the appropriate change in the index.

Proof. First we establish (ii). We know $\left[g_{i}, g_{j}\right]$ is either trivial, when $j \notin\{i-1, i+1\}$, or an odd permutation. So the commutator identities $[a b, c]=[a, c]^{b}[b, c]$ and $[a, b c]=[a, c][a, b]^{c}$ imply the first part, and the second part follows immediately using the short exact sequence (1) from Section 1.

Part (i) is now an exercise, using that $\mathrm{FAlt}^{p}=$ FAlt.

To show (iii), let $U$ be a subgroup of finite index in $\mathcal{H}_{n}$. The facts that FAlt is simple and $U$ contains a normal finite index subgroup of $\mathcal{H}_{n}$ imply that FAlt $\subset U$. Let $p$ be the smallest even integer such that $(p \mathbb{Z})^{n-1}$ is contained in the image of $U$ in the abelianization of $\mathcal{H}_{n}$. It is now clear that $U_{p}$ is contained in $U$.

Noting that $\operatorname{Com}\left(\mathcal{H}_{1}\right)=\operatorname{Aut}\left(\mathcal{H}_{1}\right)=\operatorname{Sym}$, we now characterize the commensurators of Houghton's groups.

Theorem 3.2. Let $n \geq 2$. Every commensuration of $\mathcal{H}_{n}$ normalizes $U_{p}$ for some even integer $p$. The group $N_{p}=N_{\text {Sym }}\left(U_{p}\right)$ is isomorphic to $\mathcal{H}_{n p} \rtimes\left(\mathcal{S}_{p}\right.$ 々 $\left.\mathcal{S}_{n}\right)$, and $\operatorname{Com}\left(\mathcal{H}_{n}\right)$ is the direct limit of $N_{p}$ with even $p$ under the natural embeddings $N_{p} \rightarrow N_{p q}$ for $q \in \mathbb{N}$.

Proof. Let $\phi \in \operatorname{Com}\left(\mathcal{H}_{n}\right)$. By Lemma 3.1, we can assume that $U_{p}$ is contained in the domain of both $\phi$ and $\phi^{-1}$ for some even $p$. Let $V$ be the image of $U_{p}$ under $\phi$. Then $V$ has finite index in $\mathcal{H}_{n}$ and so contains FAlt, by Lemma 3.1. However, the set of elements of finite order in $V$ equals $[V, V]$, whence $[V, V]=$ FAlt, as FAlt and FSym are not isomorphic. This means that the restriction of $\phi$ to FAlt is an automorphism of FAlt, and hence yields a homomorphism

$$
\iota: \operatorname{Com}\left(\mathcal{H}_{n}\right) \rightarrow \text { Sym. }
$$

That $\iota$ is an injective homomorphism to $\operatorname{Com}_{\text {Sym }}\left(\mathcal{H}_{n}\right)$ follows from an argument similar to the one in Proposition 2.1 applied to $g_{i}^{p}$ and six points of the form $a_{\ell}=(i, k+p \ell)$ with $\ell \in\{0,1, \ldots, 5\}$. Since the centralizer in Sym of FAlt, and hence of any finite index subgroup of $\mathcal{H}_{n}$, is trivial, the natural homomorphism from $\operatorname{Com}_{\text {Sym }}\left(\mathcal{H}_{n}\right)$ to $\operatorname{Com}\left(\mathcal{H}_{n}\right)$ mentioned above is also injective, and we conclude that $\operatorname{Com}\left(\mathcal{H}_{n}\right)$ is isomorphic to $\operatorname{Com}_{\mathrm{Sym}}\left(\mathcal{H}_{n}\right)$, and that $\iota$ is the isomorphism:

$$
\iota: \operatorname{Com}\left(\mathcal{H}_{n}\right) \rightarrow \operatorname{Com}_{\mathrm{Sym}}\left(\mathcal{H}_{n}\right) .
$$


From now on, we assume that $\phi \in \operatorname{Com}_{\text {sym }}\left(\mathcal{H}_{n}\right)$. In particular, the action of $\phi$ is given by conjugation, and our hypothesis is that $U_{p}^{\phi} \subset \mathcal{H}_{n}$. Now we can apply the argument from the proof of Theorem 2.2 to $\sigma \in U$ and $\sigma^{\phi}$ (instead of $\sigma^{\alpha}$ ). Namely, consider the $i$-th ray, and choose a $\sigma=g_{j i}^{p} \in U_{p}$. So we have $t_{i}(\sigma)=p, t_{j}(\sigma)=-p$ and zero translation elsewhere. Now, except for finitely many points, $\sigma^{\phi}$ preserves the rays and sends $x \phi$ to $x \sigma \phi$. Thus there is an infinite subset of the $i$-th ray which is sent to the same ray by $\phi$, say ray $k$. The infinite subset should be thought of as a union of congruence classes modulo $p$, except for finitely many points. We claim that no infinite subset of ray $j$ can be mapped by $\phi$ to ray $k$. This is because if it were, the infinite subset would contain a congruence class modulo $p$ (except for finitely many points) from which we would be able to produce two points $x, y$ in the support of $\sigma^{\phi}$ such that all sufficiently large positive powers of $\sigma^{\phi}$ send $x$ into ray $k$ and all sufficiently large negative powers of $\sigma^{\phi}$ send $y$ into ray $k$, and this is not possible for an element of $\mathcal{H}_{n}$. This means that $\phi$ maps an infinite subset of ray $i$ onto almost all of ray $k$ (observe that ray $k$ is almost contained in the support of $\sigma^{\phi}$ so must be almost contained in the image of the union of rays $i$ and $j$ ). Now applying similar arguments to $\phi^{-1}$ we get that $\phi$ is a bijection between rays $i$ and $k$ except for finitely many points. In fact, $\phi$ must induce bijections between the congruence classes modulo $p$ (except for finitely many points) inside those two rays.

Thus $\phi$ induces a permutation of the ray system. Again, looking at large positive powers, we deduce that $t_{k}\left(\sigma^{\phi}\right)>0$ and since the support of $\sigma^{\phi}$ is almost equal to the union of two rays, we must have $t_{k}\left(\sigma^{\phi}\right)=p$, as $\sigma$ and hence $\sigma^{\phi}$ have exactly $p$ orbits. In particular, we may deduce that $\phi$ normalizes $U_{p}$. So $U_{p}^{\phi}=U_{p}$.

In order to proceed, it will be useful to change the ray system. Specifically, each ray can be split into $p$ rays, preserving the order. This realizes $U_{p}$ as a (normal) subgroup of $\mathcal{H}_{n p}$. We say two of these new rays are equivalent if they came from the same old ray. Thus there are $n$ equivalence classes, each having $p$ elements. The group $U_{p}$ acts on this new ray system as the subgroup of $\mathcal{H}_{n p}$ consisting of all $\sigma \in$ $\mathcal{H}_{n p}$ such that $t_{i}(\sigma)=t_{j}(\sigma)$ whenever the $i$-th and $j$-th rays are equivalent. Because we have split the rays, these translation amounts can be arbitrary in $U_{p}$ (as a subgroup of $\mathcal{H}_{n p}$ ) and not just multiples of $p$. In particular, we have $U_{p}=U_{p}^{\phi} \subset \mathcal{H}_{n p}$ and the previous arguments imply that $\phi$ induces a bijection on the new ray system and sends equivalent rays to equivalent rays (since it is actually permuting the old ray system). Since $\phi$ permutes the rays, but must preserve equivalence classes, we get a homomorphism from $N_{\text {Sym }}\left(U_{p}\right)$ to the subgroup of $\mathcal{S}_{n p}$ which preserves the equivalence classes - this is easily seen to be $\left(\mathcal{S}_{p}<\mathcal{S}_{n}\right)$ and the above homomorphism is split.

As in Theorem 2.2 we now conclude that the kernel of this homomorphism is $\mathcal{H}_{n p}$ and hence we get that $N_{p}=N_{\text {Sym }}\left(U_{p}\right)$ is $\mathcal{H}_{n p} \rtimes\left(\mathcal{S}_{p} \prec \mathcal{S}_{n}\right)$.

We note that $\operatorname{Com}\left(\mathcal{H}_{n}\right)$ is not finitely generated, for if it were, it would lie in some maximal $N_{p}$. 


\section{Metric estimates for $\mathcal{H}_{n}$}

In this section we will give sharp estimates for the word length of elements of Houghton's groups. This makes no sense for $\mathcal{H}_{1}$, which is not finitely generated. As mentioned in the Introduction, the metric in $\mathcal{H}_{2}$ was described by Lehnert [2009]. In order to deal with $\mathcal{H}_{n}$ for $n \geq 3$, we introduce the following measure of complexity of an element.

Given $\sigma \in \mathcal{H}_{n}$, we define $p_{i}(\sigma)$, for $i \in \mathbb{Z}_{n}$, to be the largest integer such that $\left(i, p_{i}(\sigma)\right) \sigma \neq\left(i, p_{i}(\sigma)+t_{i}(\sigma)\right)$. Note that if $t_{i}(\sigma)<0$, then necessarily $p_{i}(\sigma) \geq\left|t_{i}(\sigma)\right|$, as the first element in each ray is numbered 1 .

The complexity of $\sigma \in \mathcal{H}_{n}$ is the natural number $P(\sigma)$ defined by

$$
P(\sigma)=\sum_{i \in \mathbb{Z}_{n}} p_{i}(\sigma)
$$

And the translation amount of $\sigma$ is

$$
T(\sigma)=\frac{1}{2} \sum_{i \in \mathbb{Z}_{n}}\left|t_{i}(\sigma)\right| .
$$

The above remark combined with (2) immediately implies $P(\sigma) \geq T(\sigma)$. It is easy to see that an element with complexity zero is trivial, and only the generators $g_{i j}$ have complexity one.

Theorem 4.1. Let $n \geq 3$ and $\sigma \in \mathcal{H}_{n}$, with complexity $P=P(\sigma) \geq 2$. Then the word length $|\sigma|$ of $\sigma$ with respect to any finite generating set satisfies

$$
P / C \leq|\sigma| \leq K P \log P,
$$

where the constants $C$ and $K$ only depend on the choice of generating set.

Proof. Since the word length with respect to two different finite generating sets differs only by a multiplicative constant, we can and will choose $\left\{g_{i j} \mid i, j \in \mathbb{Z}_{n}, i \neq j\right\}$ as the generating set to work with, and show that the statement holds with $C=1$ and $K=7$.

The lower bound is established by examining how multiplication by a generator can change the complexity. Suppose $\sigma$ has complexity $P$ and consider $\sigma g_{i j}$. It is not difficult to see that

$$
p_{k}\left(\sigma g_{i j}\right)= \begin{cases}p_{k}(\sigma)+1 & \text { if } k=i \text { and }\left(i, p_{i}(\sigma)+1\right) \sigma=(i, 1), \\ p_{k}(\sigma)-1 & \text { if } k=j,\left(j, p_{j}(\sigma)+1\right) \sigma=(j, 1), \\ p_{k}(\sigma) & \text { otherwise, } \quad \text { and }\left(j, p_{j}(\sigma)\right) \sigma=(i, 1),\end{cases}
$$

where the first two cases are mutually exclusive, as $i \neq j$. Thus $\left|P\left(\sigma g_{i j}\right)-P(\sigma)\right| \leq 1$, which establishes the lower bound. 
The upper bound is obtained as follows. Suppose $\sigma \in \mathcal{H}_{n}$ has complexity $P$. First we show by induction on $T=T(\sigma)$ that there is a word $\rho$ of length at most $T \leq P$ such that the complexity of $\sigma \rho$ is $\bar{P}$ with $\bar{P} \leq P$ and $T(\sigma \rho)=0$. The case $T=0$ is trivial. If $T>0$, then there are $i, j \in \mathbb{Z}_{n}$ with $t_{i}(\sigma)>0$ and $t_{j}(\sigma)<0$. So $T\left(\sigma g_{i j}\right)=T-1$. Moreover, $P\left(\sigma g_{i j}\right) \leq P$, because the first case of (3) is excluded, as it implies that $t_{i}(\sigma)=-p_{i}(\sigma) \leq 0$, contrary to our assumption. This completes the induction step.

We are now in the situation that $\sigma \rho \in$ FSym and loosely speaking we proceed as follows.

1. We push all irregularities into ray 0 , i.e., multiply by $\prod g_{i 0}^{p_{i}(\sigma \rho)}$.

2. We push all points back into the ray to which they belong, except for those from ray 0 which we mix into ray 1 , say.

3. We push out of ray 1 separating the points belonging to rays 0 and 1 into ray 0 and any other ray, say ray 2 , respectively.

4. We push the points belonging to ray 1 back from ray 2 into it.

These four steps can be achieved by multiplying by an element $\mu$ of length at most $4 \bar{P}$, such that $\sigma \rho \mu$ is an element which, for each $i$, permutes an initial segment $I_{i}$ of ray $i$. Notice that $\sigma \rho \mu$ is now an element of $\mathcal{H}_{n}$ which maps each ray to itself, and hence $t_{i}(\sigma \rho \mu)=0$ for all $i$.

It is clear that $\mu$ can be chosen so that the total length of the moved intervals $\sum\left|I_{i}\right|$ is at most $\bar{P}$. Finally, we sort each of these intervals using a recursive procedure, modeled on standard merge sort.

To sort the interval $I=I_{2}$ say, we push each of its points out of ray 2 and into either ray 0 if it belongs to the lower half, or ray 1 if it belongs to the upper half of $I$. If each of the two halves occurs in the correct order, then we only have to push them back into ray 2 and are done, having used $2|I|$ generators. If the two halves are not yet sorted, then we use the same "separate the upper and lower halves" approach on each of them recursively in order to sort them. In total this takes at most $2|I| \log _{2}|I|$ steps.

Altogether we have used at most

$$
P+4 \bar{P}+2 \sum_{i \in \mathbb{Z}_{n}}\left|I_{i}\right| \log _{2}\left|I_{i}\right| \leq 7 P \log _{2} P
$$

generators to represent the inverse of $\sigma$; we used the hypothesis $P \geq 2$ in the last inequality.

We note that because there are many permutations, the fraction of elements which are close to the lower bound goes to zero in much the same way as shown for Thompson's group $V$ by Birget [2004] and its generalization $n V$ by Burillo and Cleary [2010]. 
Lemma 4.2. Let $n \geq 3$. For $\mathcal{H}_{n}$ take the generating set $g_{1}, \ldots, g_{n-1}$ with $n-1$ elements. Consider the following sets:

- $B_{k}$ is the ball of radius $k$,

- $C_{k}$ is the set of elements in $\mathcal{H}_{n}$ which have complexity $P=k$,

- $D_{k} \subset C_{k}$ is the set of elements of $C_{k}$ which have word length at most $k \log _{2 n-2} k$.

Then

$$
\lim _{k \rightarrow \infty} \frac{\left|D_{k}\right|}{\left|C_{k}\right|}=0
$$

An element of complexity $P$, according to the metric estimates proved above, has word length between $P$ and $P \log P$. What this lemma means is that most elements with complexity $P$ will have word length closer to $P \log P$ than to $P$.

Proof. Observe that

$$
\frac{\left|D_{k}\right|}{\mid B_{k} \log _{2 n-2} k} \leq 1
$$

because it is a subset. Now, introduce the $C_{k}$ as

$$
\frac{\left|D_{k}\right|}{\left|C_{k}\right|} \frac{\left|C_{k}\right|}{\mid B_{k} \log _{2 n-2} k}
$$

and the proof will be complete if we show that

$$
\lim _{k \rightarrow \infty} \frac{\left|C_{k}\right|}{\mid B_{k} \log _{2 n-2} k}=\infty
$$

In $C_{k}$ there are at least $(n k-2)$ ! elements. This is because we can take a transposition of a point at distance $k$ down one of the rays with another point. Since this already ensures $P=k$, we are free to choose any permutation of the other $n k-2$ points that are at one of the first $k$ positions in each ray. And inside $\left|B_{k} \log _{2 n-2} k\right|$, counting grossly according to the number of generators, there are at most $(2 n-2)^{k \log _{2 n-2} k}=k^{k}$ elements. Now the limit becomes

$$
\lim _{k \rightarrow \infty} \frac{(n k-2) !}{k^{k}},
$$

which is easily seen to approach infinity using Stirling's formula and the fact that $n \geq 3$.

Consequentially, these estimates give an easy way to see that the group has exponential growth. We note that exponential growth also follows easily from the fact that $g_{01}$ and $g_{02}$ generate a free subsemigroup.

Proposition 4.3. Let $n \geq 3$. Then $\mathcal{H}_{n}$ has exponential growth. 
Proof. Consider a finitary permutation of complexity $P$, and observe that there are at least $P$ ! of those. By the metric estimate, its word length is at most $K P \log P$. Using again as in the previous lemma the notation $B_{k}$ for a ball, the group will have exponential growth if

$$
\lim _{k \rightarrow \infty} \frac{\log \left|B_{k}\right|}{k}>0
$$

In our case, this amounts to

$$
\lim _{P \rightarrow \infty} \frac{\log \left|B_{K P \log P}\right|}{K P \log P} \geq \lim _{P \rightarrow \infty} \frac{\log (P !)}{K P \log P}=\frac{1}{K} .
$$

\section{Subgroup embeddings}

We note that each $\mathcal{H}_{n}$ is a subgroup of $\mathcal{H}_{m}$ for $n<m$ and that our estimates together with work of Lehnert are enough to give at least quadratic distortion for some of these embeddings.

Theorem 5.1. The group $\mathcal{H}_{2}$ is at least quadratically distorted in $\mathcal{H}_{m}$ for $m \geq 3$.

Proof. We consider the element $\sigma_{n}$ of $\mathcal{H}_{2}$ which has $T\left(\sigma_{n}\right)=0$ and transposes $(0, k)$ and $(1, k)$ for all $k \leq n$. Then $\sigma_{n}$ corresponds to the word $g_{n}$ defined in Theorem 8 of [Lehnert 2009], where it is shown to have length of the order of $n^{2}$ with respect to the generators of $\mathcal{H}_{2}$ in Lemma 10 there, which are exactly the generators for $\mathcal{H}_{2}$ given in Section 1. One can easily check that $\sigma_{n}=g_{02}^{n} g_{12}^{n} g_{02}^{-n} g_{12}^{-n}$ in $\mathcal{H}_{3}$. Thus a family of words of quadratically growing length in $\mathcal{H}_{2}$ has linearly growing length in $\mathcal{H}_{3}$, which proves the theorem.

A natural, but seemingly difficult, question is whether $\mathcal{H}_{n}$ is distorted in $\mathcal{H}_{m}$ for $3 \leq n<m$. Another question, which also seems difficult, is whether $\mathcal{H}_{n}$ is distorted in Thompson's group $V$, under the embeddings mentioned in the Introduction [Röver 1999].

\section{Some quasi-isometries of $\mathcal{H}_{n}$}

Commensurations give rise to quasi-isometries and are often a rich source of examples of quasi-isometries. Here we show that the natural map from the commensurator of $\mathcal{H}_{n}$ to the quasi-isometry group of $\mathcal{H}_{n}$, which we denote by $\mathrm{QI}\left(\mathcal{H}_{n}\right)$, is an injection. That is, we show that each commensuration is not within a bounded distance of the identity. That this is an injection also follows from the more general argument of Whyte which appears as Proposition 7.5 in [Farb and Mosher 2002].

Theorem 6.1. The natural homomorphism from $\operatorname{Com}\left(\mathcal{H}_{n}\right)$ to $\mathrm{QI}\left(\mathcal{H}_{n}\right)$ is an embedding for $n \geq 2$. 
Proof. We will show that for each nontrivial $\phi \in \operatorname{Com}\left(\mathcal{H}_{n}\right)$ and every $N \in \mathbb{N}$ we can find a $\sigma \in \mathcal{H}_{n}$ such that $d\left(\sigma, \sigma^{\phi}\right) \geq N$, so none of the nontrivial images are within a bounded distance of the identity. By Theorem 3.2, we can and will view $\phi$ as a nontrivial element of $N_{p} \subset$ Sym for some even $p$.

If $\phi$ eventually translates a ray $i$ nontrivially to a possibly different ray $j$, then we let $\sigma=((i, N)(i, N+1))$, a transposition in the translated ray. The image of $\sigma$ under conjugation by $\phi$ is the transposition $\left((j, N+t)\left(j, N+t^{\prime}+1\right)\right)$, and the distance $d\left(\sigma, \sigma^{\phi}\right)$ is the length of $\sigma^{-1} \sigma^{\phi}$, which is at least $N$ since it moves at least one point at distance $N$ down one of the rays.

If $\phi$ does not eventually translate a ray but maps almost all of ray $j$ to ray $i$ with $i \neq j$, then we can show boundedness away from the identity by taking $\sigma=((j, N)(j, N+1))$. The point $(i, N)$ is fixed by $\sigma$ but is moved to $(i, N+1)$ under $\sigma^{\phi}$ ensuring that the length of $\sigma^{-1} \sigma^{\phi}$ is at least $N$.

Finally, if $\phi$ does not have the preceding two properties, then $\phi$ is a nontrivial finitary permutation. Since Houghton's group is $k$-transitive, for every $k$, we can find a $\sigma \in \mathcal{H}_{n}$ such that $\phi^{\sigma}$ has support disjoint from that of $\phi$, and at distance at least $N$ down one of the rays. Hence $\sigma^{-1} \phi^{-1} \sigma \phi=\sigma^{-1} \sigma^{\phi}$ has length at least $N$.

\section{Co-Hopficity}

Houghton's groups have been long known to be Hopfian although they are not residually finite; see [CGP 2007]. In this section we will prove that $\mathcal{H}_{n}$ is not co-Hopfian, by exhibiting a map which is injective but not surjective:

$$
\begin{gathered}
f: \mathcal{H}_{n} \rightarrow \mathcal{H}_{n}, \\
s \mapsto f(s),
\end{gathered}
$$

defined by: if $(i, n) s=(j, m)$, then

$$
(i, 2 n-1) f(s)=(j, 2 m-1) \text { and }(i, 2 n) f(s)=(j, 2 m) .
$$

It is straightforward to show that $f$ is a homomorphism. It is injective, because if $s$ is not the identity with $(i, n) s \neq(i, n)$, then $(i, 2 n) f(s) \neq(i, 2 n)$. And clearly the map is not surjective, because the permutation always sends adjacent points $(i, 2 n-1),(i, 2 n)$ to adjacent points, and a permutation which does not do this cannot be in the image.

In fact, $\mathcal{H}_{n}$ has many proper subgroups isomorphic to the whole group. The following argument was pointed out to us by Peter Kropholler.

One can well-order the ray system by taking a lexicographic order. The group $\mathcal{H}_{n}$ is then the group of all almost order-preserving bijections of the well-ordered ray system. It is then clear that the ray system minus a point is order isomorphic to 
the original ray system, which demonstrates that a point stabilizer is a subgroup isomorphic to $\mathcal{H}_{n}$.

Theorem 7.1. Houghton's groups $\mathcal{H}_{n}$ are not co-Hopfian.

\section{References}

[Birget 2004] J.-C. Birget, "The groups of Richard Thompson and complexity", Internat. J. Algebra Comput. 14:5-6 (2004), 569-626. MR 2104771 Zbl 1088.20016

[Brown 1987] K. S. Brown, "Finiteness properties of groups", J. Pure Appl. Algebra 44:1-3 (1987), 45-75. MR 885095 Zbl 0613.20033

[Burillo and Cleary 2010] J. Burillo and S. Cleary, "Metric properties of higher-dimensional Thompson's groups", Pacific J. Math. 248:1 (2010), 49-62. MR 2734164 Zbl 1236.20045

[CGP 2007] Y. de Cornulier, L. Guyot, and W. Pitsch, "On the isolated points in the space of groups", J. Algebra 307:1 (2007), 254-277. MR 2278053 Zbl 1132.20018

[Dixon and Mortimer 1996] J. D. Dixon and B. Mortimer, Permutation groups, Graduate Texts in Mathematics 163, Springer, New York, 1996. MR 1409812 Zbl 0951.20001

[Farb and Mosher 2002] B. Farb and L. Mosher, "The geometry of surface-by-free groups", Geom. Funct. Anal. 12:5 (2002), 915-963. MR 1937831 Zbl 1048.20026

[Houghton 1978] C. H. Houghton, "The first cohomology of a group with permutation module coefficients”, Arch. Math. (Basel) 31:3 (1978), 254-258. MR 521478 Zbl 0377.20044

[Lee 2012] S. R. Lee, “Geometry of Houghton's groups”, preprint, 2012. arXiv 1212.0257

[Lehnert 2009] J. Lehnert, "Some remarks on depth of dead ends in groups", Internat. J. Algebra Comput. 19:4 (2009), 585-594. MR 2536193 Zbl 1191.20027

[Röver 1999] C. E. Röver, Subgroups of finitely presented simple groups, Ph.D. thesis, University of Oxford, 1999, available at http://www.maths.nuigalway.ie/ chew/papers/thesis.shtml.

[Scott 1964] W. R. Scott, Group theory, Prentice-Hall, Englewood Cliffs, NJ, 1964. MR 0167513 Zbl 0126.04504

Received February 22, 2016.

JOSÉ BURILLO

Departament de Matemàtica Aplicada IV

EETAC-UPC

ESTEVE TERRADES 5

08860 CASTELLDEFELS

SPAIN

burillo@ma4.upc.edu

\section{SEAN CLEARY}

Department of Mathematics R8133

The City College of New York

160 Convent AVEnUE

NEW YORK, NY 10031

UNITED STATES

scleary@ccny.cuny.edu 
ARMANDO MARTINO

MATHEMATICAL SCIENCES

UNIVERSITY OF SOUTHAMPTON

UNIVERSITY ROAD

SOUTHAMPTON SO17 1BJ

UNITED KINGDOM

a.martino@soton.ac.uk

ClaAs E. RÖVER

SCHOOL of Mathematics, Statistics AND ApPlied Mathematics

NUI GALWAY

UNIVERSITY ROAD

GALWAY

IRELAND

claas.roever@nuigalway.ie 


\title{
PACIFIC JOURNAL OF MATHEMATICS
}

Founded in 1951 by E. F. Beckenbach (1906-1982) and F. Wolf (1904-1989)

$$
\text { msp.org/pjm }
$$

\section{EDITORS}

\author{
Don Blasius (Managing Editor) \\ Department of Mathematics \\ University of California \\ Los Angeles, CA 90095-1555 \\ blasius@math.ucla.edu
}

\author{
Paul Balmer \\ Department of Mathematics \\ University of California \\ Los Angeles, CA 90095-1555 \\ balmer@math.ucla.edu \\ Robert Finn \\ Department of Mathematics \\ Stanford University \\ Stanford, CA 94305-2125 \\ finn@math.stanford.edu \\ Sorin Popa \\ Department of Mathematics \\ University of California \\ Los Angeles, CA 90095-1555 \\ popa@math.ucla.edu
}

\author{
Vyjayanthi Chari \\ Department of Mathematics \\ University of California \\ Riverside, CA 92521-0135 \\ chari@math.ucr.edu \\ Kefeng Liu \\ Department of Mathematics \\ University of California \\ Los Angeles, CA 90095-1555 \\ liu@math.ucla.edu \\ Igor Pak \\ Department of Mathematics \\ University of California \\ Los Angeles, CA 90095-1555 \\ pak.pjm@gmail.com \\ Paul Yang \\ Department of Mathematics \\ Princeton University \\ Princeton NJ 08544-1000 \\ yang@math.princeton.edu
}

\section{PRODUCTION}

Silvio Levy, Scientific Editor, production@msp.org

\section{SUPPORTING INSTITUTIONS}

ACADEMIA SINICA, TAIPEI

CALIFORNIA INST. OF TECHNOLOGY

STANFORD UNIVERSITY

UNIV. OF BRITISH COLUMBIA

UNIV. OF CALIFORNIA, BERKELEY

UNIV. OF CALIFORNIA, DAVIS

UNIV. OF CALIFORNIA, LOS ANGELES

UNIV. OF CALIFORNIA, RIVERSIDE

UNIV. OF CALIFORNIA, SAN DIEGO

UNIV. OF CALIF., SANTA BARBARA
KEIO UNIVERSITY

MATH. SCIENCES RESEARCH INSTITUTE

NEW MEXICO STATE UNIV.

OREGON STATE UNIV.
Daryl Cooper

Department of Mathematics

University of California

Santa Barbara, CA 93106-3080 cooper@math.ucsb.edu

Jiang-Hua Lu

Department of Mathematics

The University of Hong Kong

Pokfulam Rd., Hong Kong

jhlu@maths.hku.hk

$$
\text { Jie Qing }
$$

Department of Mathematics

University of California

Santa Cruz, CA 95064

qing@ cats.ucsc.edu

\author{
UNIV. OF CALIF., SANTA CRUZ \\ UNIV. OF MONTANA \\ UNIV. OF OREGON \\ UNIV. OF SOUTHERN CALIFORNIA \\ UNIV. OF UTAH \\ UNIV. OF WASHINGTON \\ WASHINGTON STATE UNIVERSITY
}

These supporting institutions contribute to the cost of publication of this Journal, but they are not owners or publishers and have no responsibility for its contents or policies.

See inside back cover or msp.org/pjm for submission instructions.

The subscription price for 2016 is US $\$ 440 /$ year for the electronic version, and \$600/year for print and electronic.

Subscriptions, requests for back issues and changes of subscriber address should be sent to Pacific Journal of Mathematics, P.O. Box 4163, Berkeley, CA 94704-0163, U.S.A. The Pacific Journal of Mathematics is indexed by Mathematical Reviews, Zentralblatt MATH, PASCAL CNRS Index, Referativnyi Zhurnal, Current Mathematical Publications and Web of Knowledge (Science Citation Index).

The Pacific Journal of Mathematics (ISSN 0030-8730) at the University of California, c/o Department of Mathematics, 798 Evans Hall \#3840, Berkeley, CA 94720-3840, is published twelve times a year. Periodical rate postage paid at Berkeley, CA 94704, and additional mailing offices. POSTMASTER: send address changes to Pacific Journal of Mathematics, P.O. Box 4163, Berkeley, CA 94704-0163.

PJM peer review and production are managed by EditFLOW ${ }^{\circledR}$ from Mathematical Sciences Publishers.

PUBLISHED BY

\section{I. mathematical sciences publishers}

nonprofit scientific publishing

http://msp.org/

(C) 2016 Mathematical Sciences Publishers 


\section{PACIFIC JOURNAL OF MATHEMATICS}

Volume $285 \quad$ No. $2 \quad$ December 2016

The $\mathrm{SU}(N)$ Casson-Lin invariants for links

HANS U. BODEN and ERIC HARPER

The SU(2) Casson-Lin invariant of the Hopf link

HANS U. BODEN and CHRISTOPHER M. HERALD

Commensurations and metric properties of Houghton's groups

José BuRILlo, SEAn Cleary, ARMANDo MaRTino and ClaAs

E. RÖVER

Conformal holonomy equals ambient holonomy

ANDREAS ČAP, A. RoD GOVER, C. RoBIN GRAHAM and

MATTHIAS HAMMERL

Nonorientable Lagrangian cobordisms between Legendrian knots

ORSOLA CAPOVILLA-SEARLE and LISA TRAYNOR

A strong multiplicity one theorem for $\mathrm{SL}_{2}$

JINGSONG CHAI and QING ZHANG

The Yamabe problem on noncompact CR manifolds

PAK TUNG Ho and SeONGTAG KIM

Isometry types of frame bundles

WOUTER VAN LIMBEEK

Bundles of spectra and algebraic K-theory

JOHN A. LIND

Hidden symmetries and commensurability of 2-bridge link complements

CHRISTIAN MiLlichaP and WILLIAM WORDEN

On seaweed subalgebras and meander graphs in type $C$

DMITRI I. PANYUSHEV and OKSANA S. YAKIMOVA

The genus filtration in the smooth concordance group 